\title{
EchoGéo
}

$38 \mid 2016$

Varia

\section{Bioclimatologie des infections cutanées mycosiques à Sfax (Centre-Est de la Tunisie)}

\section{Mounir Jarraya}

\section{(2) OpenEdition \\ 12 Journals}

\section{Electronic version}

URL: https://journals.openedition.org/echogeo/14764

DOI: 10.4000/echogeo.14764

ISSN: 1963-1197

\section{Publisher}

Pôle de recherche pour l'organisation et la diffusion de l'information géographique (CNRS UMR 8586)

\section{Electronic reference}

Mounir Jarraya, "Bioclimatologie des infections cutanées mycosiques à Sfax (Centre-Est de la Tunisie)", EchoGéo [Online], 38 | 2016, Online since 13 December 2016, connection on 31 July 2021. URL: http://journals.openedition.org/echogeo/14764 ; DOI: https://doi.org/10.4000/echogeo.14764

This text was automatically generated on 31 July 2021.

EchoGéo est mis à disposition selon les termes de la licence Creative Commons Attribution - Pas d'Utilisation Commerciale - Pas de Modification 4.0 International (CC BY-NC-ND) 


\title{
Bioclimatologie des infections cutanées mycosiques à Sfax (Centre- Est de la Tunisie)
}

\author{
Mounir Jarraya
}

\section{Introduction}

La relation entre l'environnement physique et la variation spatio-temporelle des pathologies infectieuses constitue depuis longtemps un thème de recherche riche en approches diverses (Ehrlich et al., 1970 ; Saad Hussein et al., 2011). La localisation géographique peut favoriser l'apparition de certaines pathologies infectieuses (Morel, 2013). La variation saisonnière de certaines maladies infectieuses a permis de suggérer une relation avec l'enveloppe atmosphérique qui commande leur propagation (Jarraya, 2014 ; Guo-Zheng et al., 2015). Partout dans le monde, et sous divers contextes climatiques, les maladies cutanées représentent une part importante dans le paysage épidémiologique infectieux. Par exemple, plus de 14 millions de consultations sont enregistrées chaque année aux États-Unis en raison des infections cutanées, avec plus de 600000 hospitalisations (3,7\% des hospitalisations en urgence) (Phoenix et al., 2012). Le nombre d'hospitalisations pour de telles infections a augmenté de $29 \%$ entre 2000 et 2004 (Edelsberg et al., 2009). Le climat chaud présent durant toute l'année et les précipitations abondantes peuvent favoriser la reproduction des agents pathogènes à l'intérieur et en dehors des organismes biologiques (Lakshmipathy et Kannabiran, 2010). Les maladies cutanées sont moins fréquentes sous climat tempéré, en partie en raison d'une saison froide durant laquelle la population d'insectes et les agents pathogènes associés hibernent (Dowell, 2001). Ces agents pathogènes désignent tout élément microscopique potentiellement dangereux (bactéries, virus, champignons), qui peut être transmis directement ou par vecteur et provoquer des atteintes à la surface cutanée. 
Les mycoses cutanées sont parmi les pathologies infectieuses communautaires, qui se diffusent rapidement dans la population et qui peuvent constituer une porte d'entrée à d'autres infections graves (Gardner-Medwin et al., 2001 ; Besancenot et al., 2004 ; Simka, 2010). Elles constituent de ce fait une gêne pour les personnes affectées et un enjeu de santé publique. Les mycoses cutanées, provoquées par des champignons microscopiques, sont le plus souvent bénignes mais gênantes, à cause des démangeaisons qu'elles entraînent (Lakshmipathy et Kannabiran, 2010). Toutes les parties du corps peuvent être touchées par les mycoses, mais celles caractérisées par la chaleur et l'humidité, comme les pieds et les plis sont les plus vulnérables. Deux principaux types de champignons peuvent être à l'origine des mycoses cutanées. Les dermatophytes, qui se transmettent soit par un animal ou une personne contaminés, soit par l'intermédiaire des sols humides. Les levures (surtout le Candida), qui sont naturellement présentes dans l'organisme et qui, dans certaines conditions (humidité, stress, prise de médicament...), peuvent entraîner une infection (Saint-Germain, 2012). Les symptômes d'une mycose cutanée consistent en une rougeur locale, accompagnée de démangeaisons et de petites peaux sèches en surface (Bouguerra et al., 2004). Dans cet ensemble pathologique "mycoses cutanées ", nous distinguons plusieurs infections comme la Dermatophytie, l'Herpes-circiné, l'Intertrigo, la Candidose et le Pityriasis versicolor... et chacune de ces maladies touche différemment la peau et se caractérise par une symptomatologie spécifique.

Dans le cadre de leur analyse de la relation entre climat, eau et santé au Sahel ouestafricain, Besancenot et al. (2004) ont confirmé l'effet de l'environnement extérieur sur la fréquence des pathologies cutanées. Ils ont attribué la forte fréquence des dermatoses comme la miliaire rouge - qui est très irritante - à une rétention élevée de la sueur. Celle-ci est le résultat de la thermolyse, un mécanisme refroidissant déclenché par le corps humain afin de rétablir son homéothermie face une ambiance chaude et humide. Il a été montré également que la faible humidité de l'air associée à l'exposition au rayonnement solaire peut favoriser le vieillissement cutané. Les symptômes sont variés et présentent des pathologies cutanées diversifiées comme la kératodermie, qui se caractérise par une peau épaisse et profondément fissurée ou la xérodermie, marquée par une peau sèche avec desquamation cendreuse (Besancenot et al., 2004). De telles formes d'atteintes de la surface cutanée favorisent l'apparition des signes mycosiques.

Compte tenu du rôle capital joué par la peau en tant qu'interface avec l'environnement extérieur et dans la régulation thermique corporelle (Geyfman et Andersen, 2009; Xhauflaire-Uhoda et al., 2011), plusieurs altérations de ses fonctions peuvent survenir. Ces altérations peuvent toucher certains biorythmes internes comme la température des doigts (Gardner-Medwin et al., 2001) ou se manifester sous des formes pathologiques, c'est-à-dire à travers des maladies cutanées, dont les fréquences varient d'une saison à l'autre. La variation saisonnière des maladies cutanées a été analysée dans plusieurs études (Dowell, 2001; Saad Hussein et al., 2011) qui montrent l'implication de l'environnement atmosphérique extérieur (la température et l'humidité de l'air) dans la survenue de ces pathologies (Tang, 2009). Les mycoses cutanées ont été mises en relation avec des éléments du contexte climatique (Besancenot et al., 2011, El-Herte et al., 2012). Par exemple, l'incidence des dermatomycoses a été corrélée aux situations thermiques et hygrométriques dans la ville de Karatchi (Pakistan) (Faruqi et al., 1987). Et il est démontré qu'une forte humidité associée à une température élevée est responsable de l'augmentation de l'incidence des 
dermatomycoses au cours de la phase de mai-août. Piérard-Franchimont et al., (2012) ont souligné l'impact du contexte thermique saisonnier sur la fréquence des ulcères veineux des jambes. Ils ont montré que le froid hivernal favorise l'affection par les ulcères, alors que la chaleur estivale contribue à leur aggravation. De même, le vieillissement accentue la vulnérabilité de la surface cutanée et la rend plus sensible aux effets de la température extérieure (Klode et al., 2011).

En Tunisie, les études cliniques dans le milieu hospitalier se sont intéressées aux infections cutanées (Bouguerra et al., 2004 ; Ben Salem et al., 2011). Cependant, l'éventuelle implication du contexte climatique dans l'explication d'une telle situation épidémiologique est peu étudiée. Une précédente étude réalisée dans la ville de Sfax a montré une forte fréquence automnale des cas d'érysipèle associée à une chaleur et une humidité élevées (Jarraya et al., 2005).

La particularité qui nous intéresse réside dans le fait que les infections mycosiques se développent davantage dans un contexte climatique chaud et humide. Cela nous permet de suggérer un lien possible entre cette fréquence élevée de la morbidité mycosique et la thermo-hygrométrie de l'atmosphère aussi bien au printemps mais surtout en automne. Ce présent travail vise à analyser, pour la période 2009-2013, l'effet de l'environnement atmosphérique sur la fréquence des consultations pour mycoses, autrement dit la concordance climato-pathologique pendant ces deux périodes de l'année à Sfax, en mettant l'accent sur l'effet de la thermo-hygrométrie printanière et automnale dans l'accentuation de la vulnérabilité de la population à ces infections cutanées.

Par ailleurs, l'analyse de la problématique indiquée ci-dessus implique la connaissance du contexte géographique, physique et humain de la zone d'étude. Sfax, une ville littorale qui se situe sur une plaine longeant la côte orientale de la Tunisie (illustration 1) abrite actuellement 650000 habitants environ et connaît une forte concentration de la population et des activités économiques. Le développement de l'agriculture dans son arrière-pays et l'installation des industries diversifiées sur la côte remontent, certes, à l'époque coloniale. Mais cette tendance s'est accentuée à la veille de l'Indépendance avec le développement d'un secteur tertiaire florissant (en particulier la santé et l'enseignement), qui a permis à Sfax de rayonner sur le centreouest et le sud tunisien (Jarraya et Beltrando, 2013). 
Illustration 1 - Localisation géographique de la zone d'étude

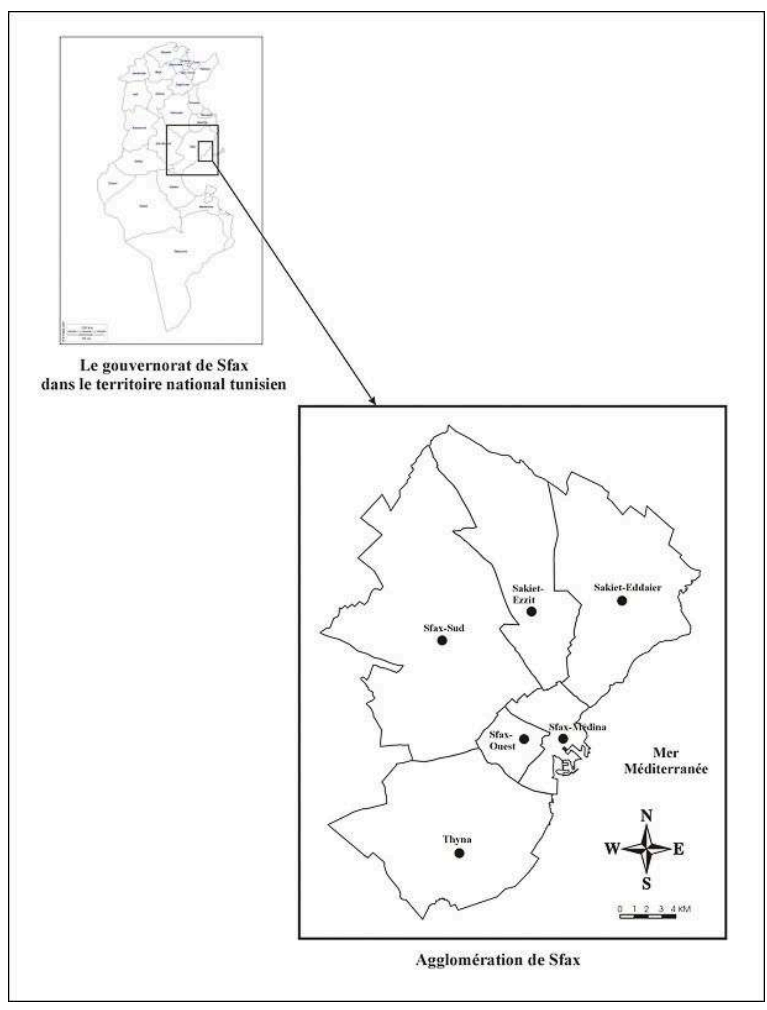

L'ouverture sur la mer a permis à cette ville, jouissant d'un climat méditerranéen semiaride, de bénéficier de son rôle régulateur de la température de l'air en hiver et en été. Ce rôle modérateur joué par la mer n'exclut pas cependant des phénomènes thermiques extrêmes (fraîcheur intense en hiver et chaleur aigüe en été) fréquents, qui conduisent à des ambiances difficiles et dangereuses pour le bien-être et la santé de l'Homme. La localisation littorale de la ville de Sfax explique l'humidité de l'air élevée en permanence. Bien qu'elles soient les saisons les plus pluvieuses durant toute l'année, l'automne et l'hiver connaissent une forte variabilité au niveau de leurs totaux pluviométriques qui traduit une probable tendance à l'aridité au fil des décennies.

Notre travail porte essentiellement sur l'analyse des situations climatiques spécifiques printanière et automnale influençant l'ambiance bioclimatique. Et nous cherchons à étudier en particulier la composante thermo-hygrométrique, qui commande également l'activité des agents pathogènes aboutissant à l'apparition des formes morbides infectieuses cutanées. Ce qui justifie cette présentation assez brève du contexte climatique

\section{Données et Méthodes}

\section{Variables relatives aux infections mycosiques}

Notre étude est rétrospective, elle s'étale sur 5 ans (2009-2013) et repose sur des données épidémiologiques obtenues de deux sources distinctes :

- données mensuelles sur les cas d'infections cutanées mycosiques colligés par la consultation du service de la dermatologie du Centre Hospitalo-Universitaire (CHU) Hédi Chaker de Sfax. 
L'ensemble pathologique étudié regroupe les infections cutanées mycosiques suivantes: mycoses des ongles, intertrigo, dermatophytie et pityriasis versicolor.

- données des consultations journalières enregistrées par 29 Centres de Santé de Base (CSB) (appelés aussi dispensaires) dans l'agglomération de Sfax. À l'inverse des données hospitalières, celles relevant de la Santé de Base n'offrent aucune information sur le type de la mycose. Ces données sont obtenues auprès de la Direction Régionale de la Santé Publique à Sfax (DRSP). Les CSB représentent la première ligne de recours dans le système de soin public tunisien. Les CSB offrent les soins ambulatoires aux habitants (Jarraya, 2012 ; Jarraya et Beltrando, 2013). Dans l'agglomération de Sfax, $60 \%$ de la population totale fréquente les structures de soins publiques (Ben Jemaâ, 2004).

En raison du refus des médecins libéraux de nous transmettre des données, seule la partie de la population, qui fréquente les structures des soins publics et plus précisément sur le service hospitalier de la dermatologie et les dispensaires a été étudiée.

\section{Variables relatives aux conditions climatiques (température et humidité relative)}

Les informations climatiques sont définies par la température de l'air $\left(\mathrm{t}^{\circ}\right.$, température moyenne $\left({ }^{\circ} \mathrm{C}\right)$ tri-horaire) et l'humidité relative ( $\mathrm{U} \%$, humidité relative moyenne trihoraire) mesurées par la station synoptique Sfax El-Maou de l'Institut National de la Météorologie (INM) installée dans l'aéroport international Sfax-Thyna.

Les données météorologiques de la température et de l'humidité relative de l'air constituent la base de l'indice Humidex (Heat Index) (HI), qui a été développé et utilisé au Canada pendant la saison chaude depuis 1979 (Masterson et Richardson, 1979) (illustration 2). L'indice Humidex est calculé en utilisant un outil qui est basé sur un algorithme disponible via les sites http://www.meteo-mussidan.fr/hum.php, http:// www.meteolafleche.com et http://www.ohcow.on.ca/menuweb/ heat_stress_calculator.htm. 
Illustration 2 - Calcul de l'indice Humidex en fonction de la température $\left({ }^{\circ} \mathrm{C}\right)$ et de l'humidité relative de l'air (\%)

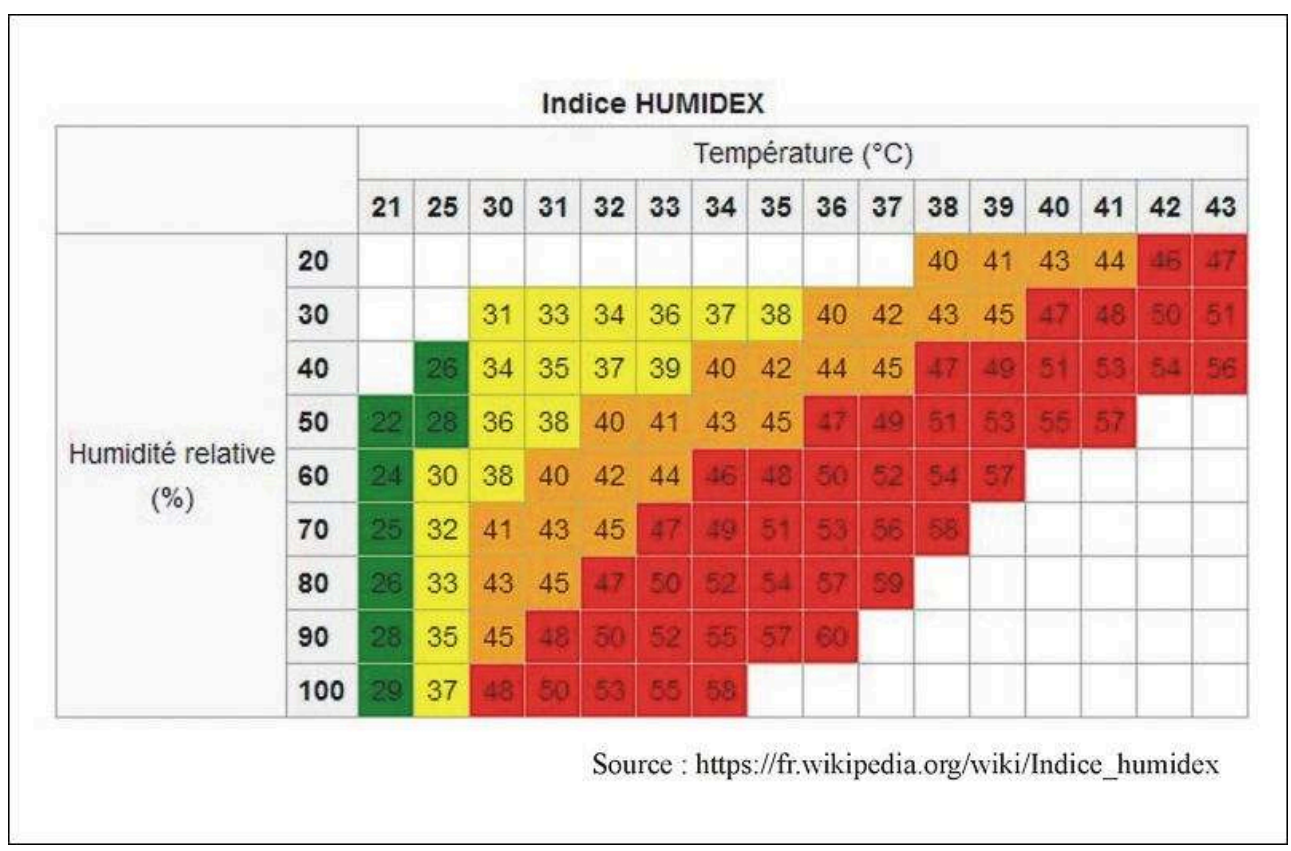

Source : https://fr.wikipedia.org/wiki/indice_humidex

Cet indice permet de distinguer des types d'ambiances associés aux fortes chaleurs. À partir d'un $\mathrm{HI}$ égal ou supérieur à $30^{\circ} \mathrm{C}$, l'ambiance tend à l'inconfort (illustration 3). Le terme mode n'a aucune signification statistique, mais exprime ici la façon dont l'organisme humain réagit face à un niveau thermo-hygrométrique présent dans l'ambiance extérieure.

Illustration 3 - Classes de l'indice Humidex (HI) et leurs modes associés

\begin{tabular}{|l|l|}
\hline $\begin{array}{l}\text { Valeurs de l'indice } \\
\text { Humidex }(\mathrm{HI})\end{array}$ & Mode exprimé \\
\hline $20<\mathrm{HI}<29$ & Sensation de bien-être \\
\hline $30<\mathrm{HI}<34$ & Sensation de malaise plus ou moins grand \\
\hline $35<\mathrm{HI}<39$ & $\begin{array}{l}\text { Sensation de malaise assez grand. Prudence, ralentir certaines } \\
\text { activités en plein air. }\end{array}$ \\
\hline $40<\mathrm{HI}<45$ & Sensation de malaise généralisée. Danger, éviter les efforts. \\
\hline $46<\mathrm{HI}<53$ & Danger extrême. Arrêt de travail dans nombreux domaines. \\
\hline
\end{tabular}

Source : http://www.meteo-mussidan.fr/hum.php

C'est durant la journée que l'ambiance thermo-hygrométrique peut avoir des effets négatifs sur le bien-être de l'organisme humain en raison de la forte chaleur et de l'humidité. Une telle ambiance offre également les conditions favorables pour une forte activité des champignons responsables des mycoses cutanées. Par conséquent, nous 
observons une exposition accrue de la population à ces agents pathogènes en raison d'une ambiance inconfortable et ce d'autant plus que les individus sont le plus souvent en dehors de leurs maisons. Nous avons donc sélectionné parmi les données climatiques (de la température et de l'humidité relative) tri-horaires, celles de $9 \mathrm{~h}, 12 \mathrm{~h}$ et $15 \mathrm{~h}$, pour calculer l'indice Humidex (HI) moyen diurne. En nous basant sur cette moyenne diurne d'indice Humidex, nous avons classé les jours inconfortables en deux phases, qui correspondent aux deux pics de morbidité d'infections mycosiques cutanées (en avril-mai et septembre-octobre) selon les modes exprimés dans le tableau (illustration 3). Ces modes présentés selon chaque intervalle de l'indice Humidex caractérisent l'état du corps humain (confort ou inconfort) en fonction du niveau de la thermo-hygrométrie de l'ambiance biométéorologique. Nous avons qualifié ces jours inconfortables «de jours à risques ", car en présentant des HI moyens diurnes élevés, ils se caractérisent par la prédominance d'ambiances thermo-hygrométriques favorables à l'apparition de mycoses. L'analyse des répercussions d'une thermohygrométrie élevée, et donc des niveaux de confort différents, sur la morbidité mycosique enregistrée permet d'éclaircir cette association météo-pathologique expliquant les deux pics observés à travers les consultations médicales à Sfax.

Deux échelles temporelles (bioclimatique et biométéorologique) sont utilisées dans notre approche traduisant le passage du général au particulier, c'est-à-dire du " bioclimatique » au " biométéorologique ». "L'intérêt de notre étude consiste à mettre en question cette échelle temporelle et son adéquation avec la représentativité de l'état réel de l'ambiance biométéorologique ainsi que sa variabilité » (Jarraya, 2012). Cet affinement d'échelles temporelles permet de zoomer et de déceler l'effet de la thermohygrométrie de l'ambiance biométéorologique ainsi que ses degrés de confort sur la morbidité mycosique. Si les caractéristiques générales de l'ambiance bioclimatique sont dégagées à l'échelle mensuelle et saisonnière pour comprendre une éventuelle relation avec la montée de la morbidité mycosique, l'échelle journalière et la définition des «jours à risques » ont le mérite de montrer l'impact de la variabilité de l'inconfort de l'ambiance biométéorologique sur la fréquence des consultations pour mycoses cutanées.

\section{Prévalence des infections mycosiques cutanées}

L'analyse des infections mycosiques cutanées repose sur les données recueillies auprès d'un service hospitalier et dans les 29 Centres de Santé de Base de l'agglomération. Le service de dermatologie du CHU Hédi Chaker a enregistré une moyenne annuelle de 529 consultations pour des cas d'infections mycosiques cutanées durant la période 2009-2013. Cet effectif varie de 455 cas en 2012 à 626 cas en 2013. Au niveau des Centres de Santé de Base, le nombre annuel moyen de consultations pour infections mycosiques est de 1584 cas. Ce chiffre varie de 1469 cas en 2011 à 1941 cas en 2013. De ce fait, le nombre annuel moyen enregistré, toutes structures sanitaires confondues (CHU et Centres de Santé de Base), est de 2113 cas durant la période 2009-2013.

La part moyenne des infections mycosiques dans l'ensemble des pathologies cutanées est non négligeable, puisqu'elle est de l'ordre de 14,5\% durant la période 2009-2013 pour l'ensemble des données de l'étude (hospitalier et centres de santé). Cette part varie de $13 \%$ en 2013 à $16 \%$ en 2010. 
L'ensemble des pathologies cutanées étudiées regroupe des infections mycosiques cutanées variées. Les données hospitalières obtenues du service de dermatologie montrent que la part de la mycose des ongles est prédominante, puisqu'elle représente $41 \%$ contre $17 \%$ pour le pityriasis versicolor et $21 \%$ pour l'intertrigo et pour la dermatophytie, durant la période 2009-2013.

\section{Un régime saisonnier?}

L'analyse des séries temporelles des consultations pour infections mycosiques cutanées laisse apparaître un rythme saisonnier spécifique. Compte tenu des spécificités des données collectées au niveau hospitalier et au niveau des Centres de Santé de Base, il est possible que cette analyse permette d'identifier certaines caractéristiques du régime saisonnier de la morbidité mycosique à Sfax que ce soit au niveau de l'effectif total ou par maladie.

$\mathrm{Au}$ niveau hospitalier, la répartition saisonnière des consultations pour infections mycosiques cutanées, toutes causes confondues, est marquée par une relative augmentation printanière mais surtout par une prédominance automnale. En effet, l'illustration 4 montre que le service de la dermatologie a enregistré 144 cas au printemps contre 123 cas en hiver sur la période 2009-2013, soit un accroissement de $9 \%$. Ce rythme de croissance s'accroît durant la phase été-automne (42,6\%), puisque la morbidité mycosique est passée de de 134 à 191 cas.

Illustration 4 - Variation saisonnière moyenne des consultations mycosiques (2009-2013)

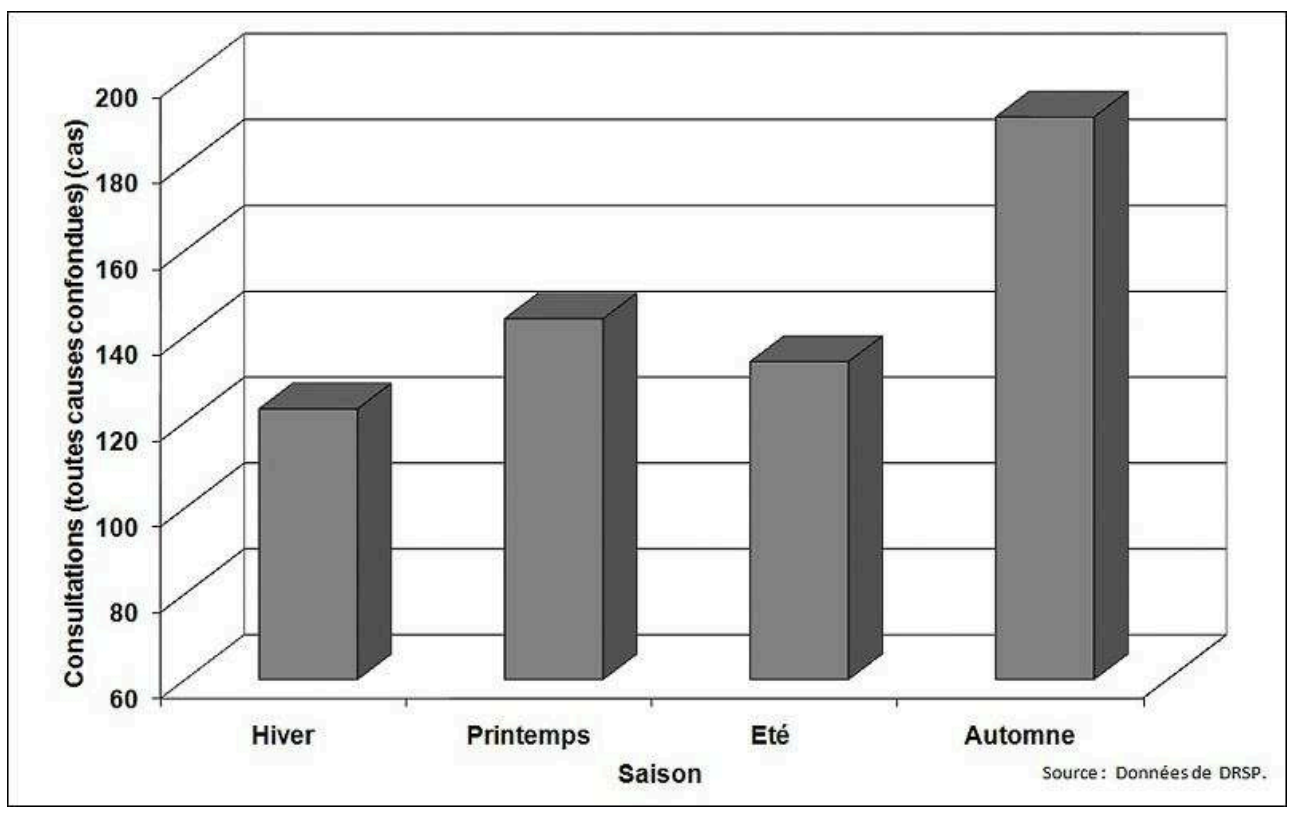

Source : Données de la DRSP.

À l'échelle mensuelle (illustration 5), l'analyse des consultations pour ces infections (toutes causes confondues), sur la période 2009-2013, montre deux pics : le premier, le moins important, survient en mai (54 cas) tandis que le second, le plus important, se présente en octobre ( 72 cas) (illustration 5$)$. Ce même rythme caractérise les consultations enregistrées dans les Centres de Santé de Base, puisque que nous 
constatons un premier pic printanier en mai (150 cas) et un deuxième pic automnal en septembre (204 cas).

L'apparition du pic automnal (en septembre) enregistré dans les consultations réalisées dans les Centres de Santé de Base est plus précoce que dans le domaine hospitalier (en octobre). Le fait que les CSB représentent le premier recours de la population peut être une explication. Ceux-ci centralisent toutes les demandes de soins avant le transfert du patient à l'hôpital.

Illustration 5 - Variation mensuelle des consultations mycosiques et de l'indice Humidex moyen (2009-2013)

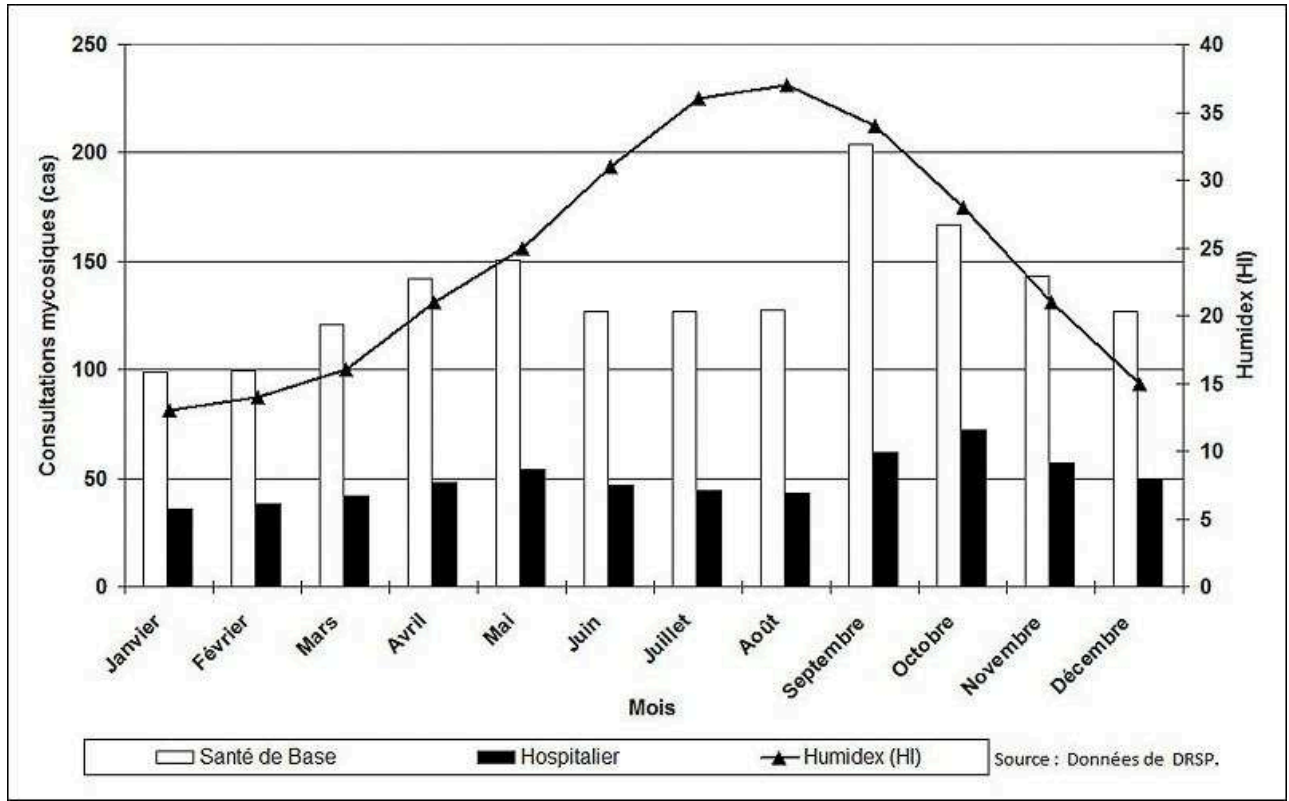

Source : Données de la DRSP.

Ce rythme saisonnier est confirmé par l'illustration 6 qui montre pour les quatre pathologies étudiées deux pics : un au printemps et un en automne. La répartition mensuelle du nombre de consultations montre une prédominance automnale : $54 \%$ des cas de Pityriasis versicolor et $71 \%$ des cas de Dermatophytie sont enregistrés en automne. Le pic automnal des consultations est enregistré en octobre pour toutes les maladies, c'est-à-dire un mois après les consultations aux CSB. En effet, les CSB sont les premiers recours de « la première vague » des affectés dès l'apparition des maladies (en août et surtout en septembre). Les répercussions sur le domaine hospitalier apparaissent plus tardivement (en octobre). Cependant, l'écart minime entre septembre et octobre au niveau des consultations pour mycose des ongles ( 1 cas), pityriasis versicolor ( 0 cas), la dermatophytie ( 1 cas) voire les 4 cas d'intertrigo, permet de parler d'un pic à double battons (illustration 6). 


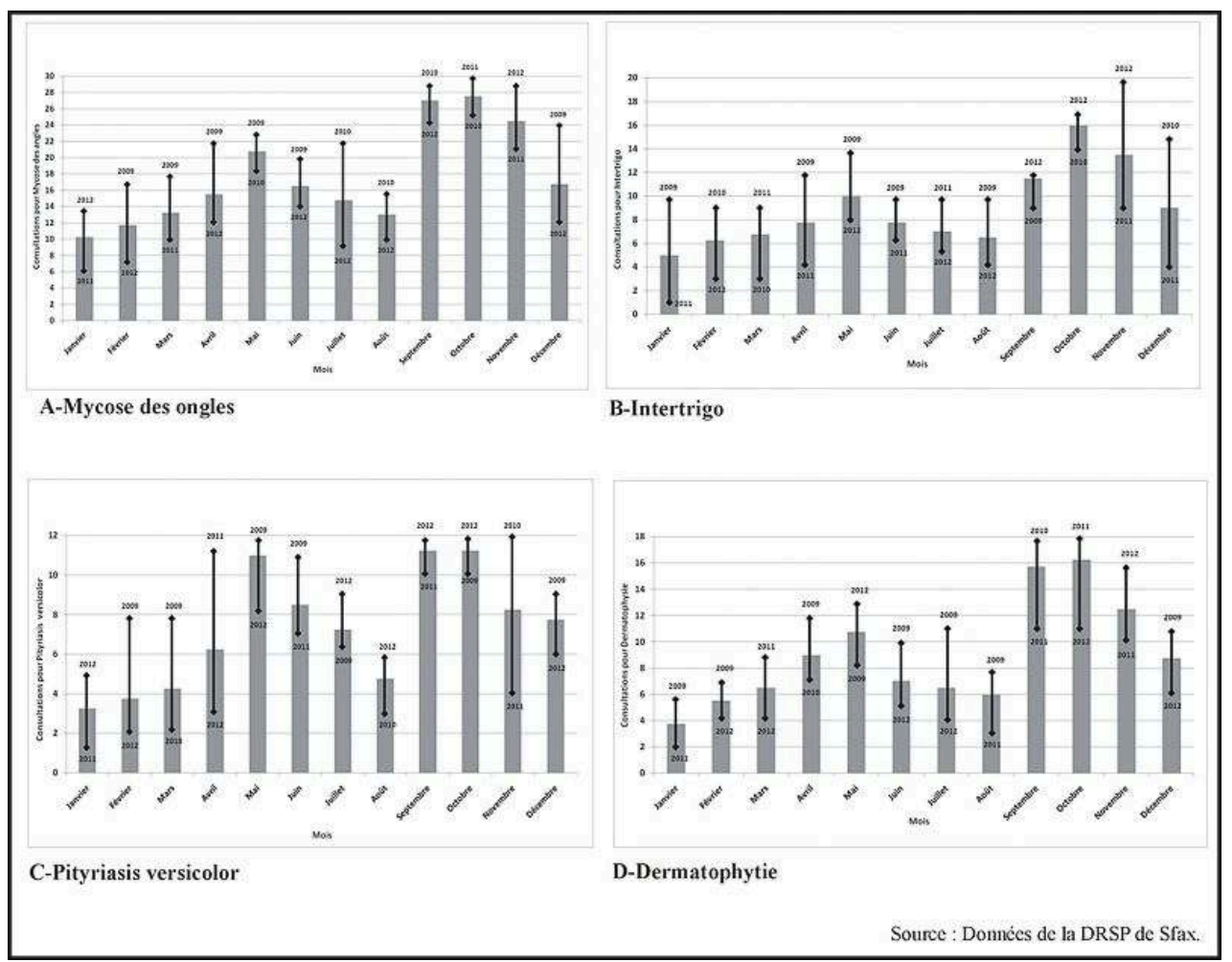

Source : Données de la DRSP

Les caractéristiques saisonnières de la morbidité mycosique conduisent à s'interroger sur le contexte climatique printanier et automnal, en particulier thermohygrométrique, et sur son impact potentiel dans la fréquence des consultations pour infections mycosiques cutanées dans les Centres de la Santé de Base à Sfax.

\section{Aspects climato-pathologiques des pics printanier et automnal}

Bien que les deux pics de la morbidité mycosique n'aient pas le même degré d'importance, nous suggérons que l'environnement atmosphérique exprimé ici par sa thermo-hygrométrie, puisse en partie expliquer l'augmentation des consultations pour mycose au printemps et en particulier durant l'automne.

\section{Le pic printanier de la morbidité mycosique : la part de la thermo- hygrométrie}

L'observation du régime mensuel de la morbidité mycosique à Sfax permet de constater une augmentation des consultations en fin d'hiver (100 cas en février), qui atteint son pic en mai ( 150 cas), dans le secteur de Santé de Base (illustration 5). Cette phase printanière est caractérisée par un adoucissement thermique, qui est associé à une humidité assez élevée et qui s'exprime par un accroissement progressif de HI de 16 en mars à 25 en mai, à l'origine d'une recrudescence relative de la morbidité mycosique de 
$24 \%$ entre ces deux mois. Ce premier constat d'une éventuelle relation entre l'augmentation concomitante de l'indice Humidex et de la morbidité mycosique observée à l'échelle mensuelle sera examiné, en adoptant une échelle plus fine (journalière) et en intégrant le facteur du confort thermo-hygrométrique.

La relation entre la thermo-hygrométrie de l'atmosphère et la morbidité mycosique enregistrée par les Centres de Santé de Base à Sfax sera examinée en déterminant tout d'abord l'importance des jours inconfortables (qualifiés ici des jours à risque) et ensuite les consultations qui en découlent.

\section{Définition des jours à risque}

L'analyse de la moyenne diurne de l'indice Humidex HI montre une tendance nette à l'augmentation en avril et surtout en mai. Le mois d'avril se caractérise par des HI diurnes moyennes le plus souvent supérieures à 22 et dépassant fréquemment le seuil de 26 durant la deuxième moitié de mai (illustration 7). Cette augmentation de la thermo-hygrométrie peut favoriser l'activité des champignons responsables des mycoses. L'adoucissement thermique post-hivernal associé à une humidité persistante et élevée (entretenue également par la localisation côtière de Sfax) explique une thermo-hygrométrie printanière favorable. Par ailleurs, l'association du niveau de confort présent dans l'atmosphère à celui de la morbidité mycosique correspondant permet de dégager certains aspects météopathologiques durant avril et mai.

Illustration 7- Moyenne quotidienne diurne de l'indice Humidex (HI) en avril et mai (2009-2013)

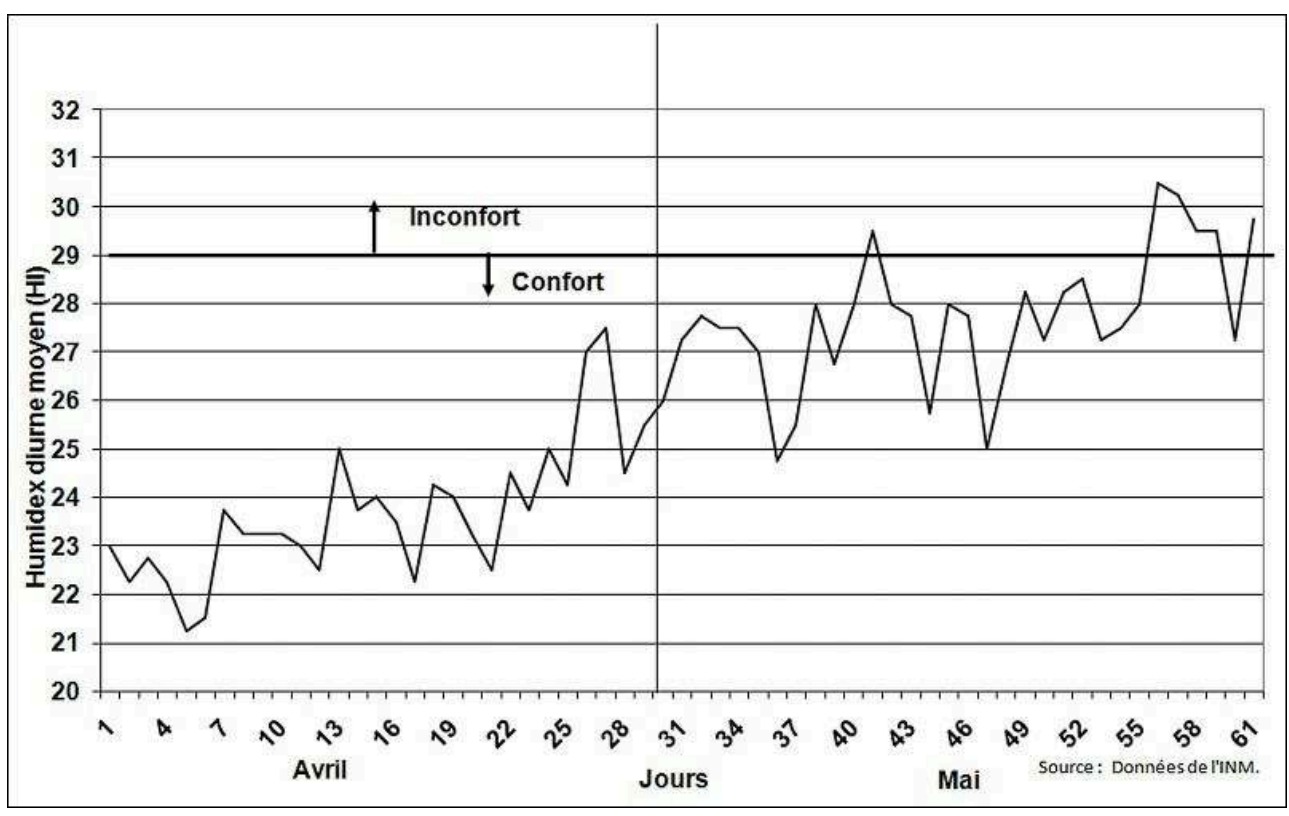

Source : Données de l'INM.

La définition des jours à risque se base sur l'indice Humidex et montre que pour un individu, le risque de l'infection par mycose s'accroît avec l'augmentation de l'inconfort, reflet de la chaleur et de l'humidité qui se maintiennent dans l'air à des niveaux élevés. En effet, les moyennes diurnes de HI indiquent que la part des jours inconfortables s'accroît de 4,7\% en avril (3 jours) à 33,4\% en mai (11 jours) sur la 
période 2009-2013. C'est le mode exprimant un malaise plus ou moins grand $(30<\mathrm{HI}<34)$ qui prédomine durant les jours inconfortables (illustration 8 ).

Illustration 8 - Variation inter-annuelle de la part des jours exprimant des modes inconfortables en avril et mai

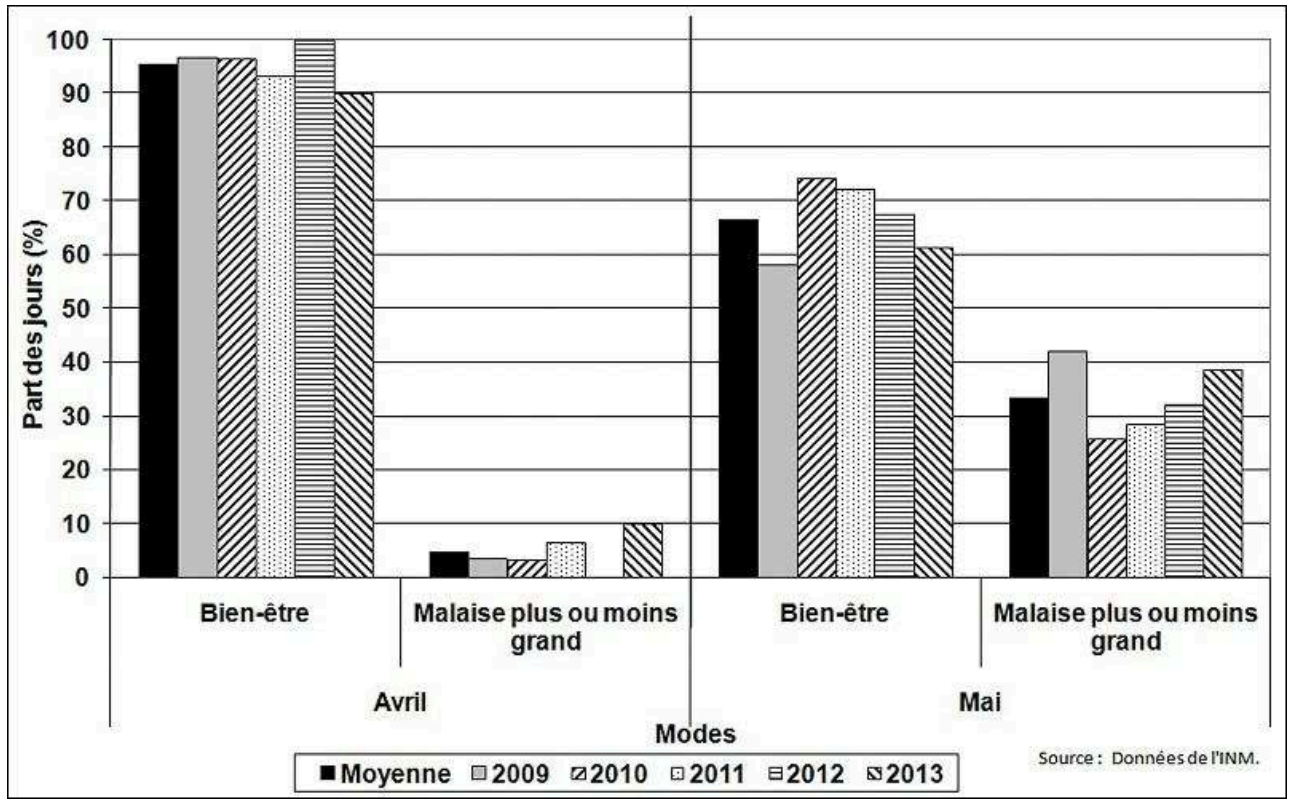

Source : Données de l'INM.

Les deux modes, confortable et inconfortable, observés font l'objet d'une variation inter-annuelle plus nette en mai qu'en avril. La part des jours confortables exprimant le bien-être $(20<\mathrm{HI}<29$ varie de $58 \%$ en 2009 à $74,2 \%$ en 2010 alors que celle des jours inconfortables exprimant un malaise plus ou moins grand varie de $42 \%$ en 2009 à $25,8 \%$ en 2010 (illustration 8);

\section{Aspects météopathologiques associés}

Bien que le mode confortable exprimant le bien-être prédomine en avril (95,3\% du nombre total de jours sur la période $2009-2013$ et $100 \%$ en 2012), des jours inconfortables exprimant un malaise plus ou moins grand peuvent apparaître ( $10 \% \mathrm{du}$ nombre total des jours en 2013) et influencer la morbidité mycosique. La moyenne diurne de l'indice Humidex (HI) des jours qui expriment un malaise plus ou moins grand est de l'ordre de 30,5, alors qu'elle est de 23,4 au niveau des jours qui expriment un bien-être. L'inconfort influe sur la morbidité mycosique, qui croît de 3 à 6 cas entre les deux modes en avril (illustration 9). En mai, l'augmentation de l'indice Humidex influence fortement les moyennes de HI diurne entre les deux modes, dans la mesure où celles-ci passent de 25,7 pour les jours exprimant un bien-être à 32 pour les jours exprimant un malaise plus ou moins grand. L"accroissement de la part des jours inconfortables $(42 \%$ en 2009) et de leur moyenne de HI diurne conduit à une augmentation relative de la morbidité mycosique, allant de 5 cas à 10 cas en mai (illustration 9). 
Illustration 9 - Consultations mycosiques associées aux moyennes de $\mathrm{HI}$ des jours exprimant un bien-être et un malaise plus ou moins grand en avril et mai

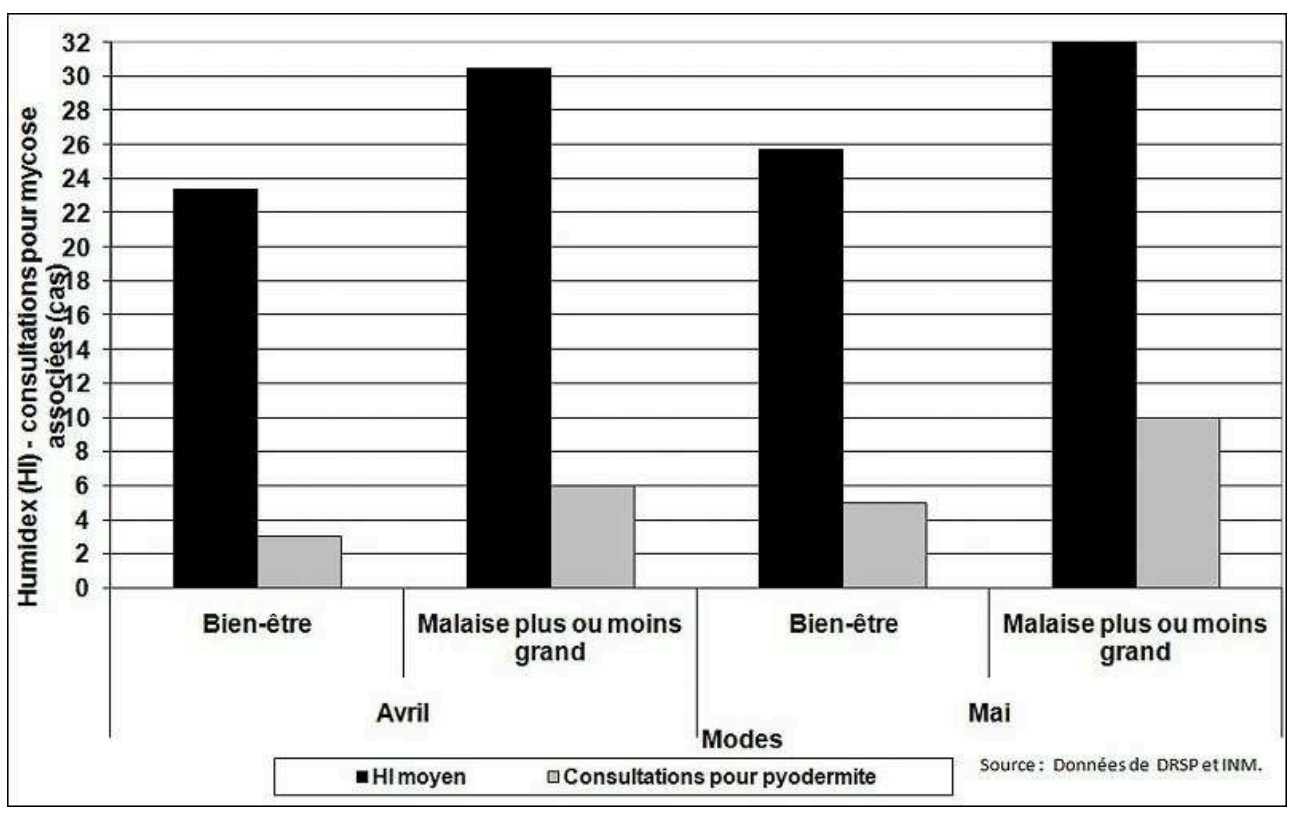

Sources : Données de la DRSP et de I'INM.

La prédominance du mode confortable exprimant le bien-être explique donc la fréquence plus ou moins faible des consultations pour mycoses au printemps. Cependant, le pic observé en mai s'explique par l'accroissement du mode inconfortable qui exprime un mode thermo-hygrométrique non seulement gênant pour le bien-être des individus mais aussi favorable à l'activité des champignons responsables des infections mycosiques.

\section{Le pic automnal de la morbidité mycosique : une forte implication de l'humidité}

L'analyse des consultations moyennes mensuelles montre une augmentation en fin d'été (127 cas en août) et un pic en septembre (204 cas) malgré la diminution relative de l'indice Humidex HI, respectivement de 37 à 34 (illustration 10). Cette fréquence élevée de la morbidité mycosique peut être due une configuration bioclimatique marquée par la montée de l'humidité et de la chaleur estivale associée à une forte activité de l'agent pathogène, favorisée par une telle situation bioclimatique. La persistance de la chaleur estivale en automne s'explique par l'extension de la saison chaude à Sfax, qui s'étale sur six mois (de mai en octobre) (Dahech et Bouaziz, 2010 ; Dahech, 2014). 
Illustration 10 - Moyenne quotidienne diurne de l'indice Humidex $\mathrm{HI}$ en septembre et octobre (2009-2013)

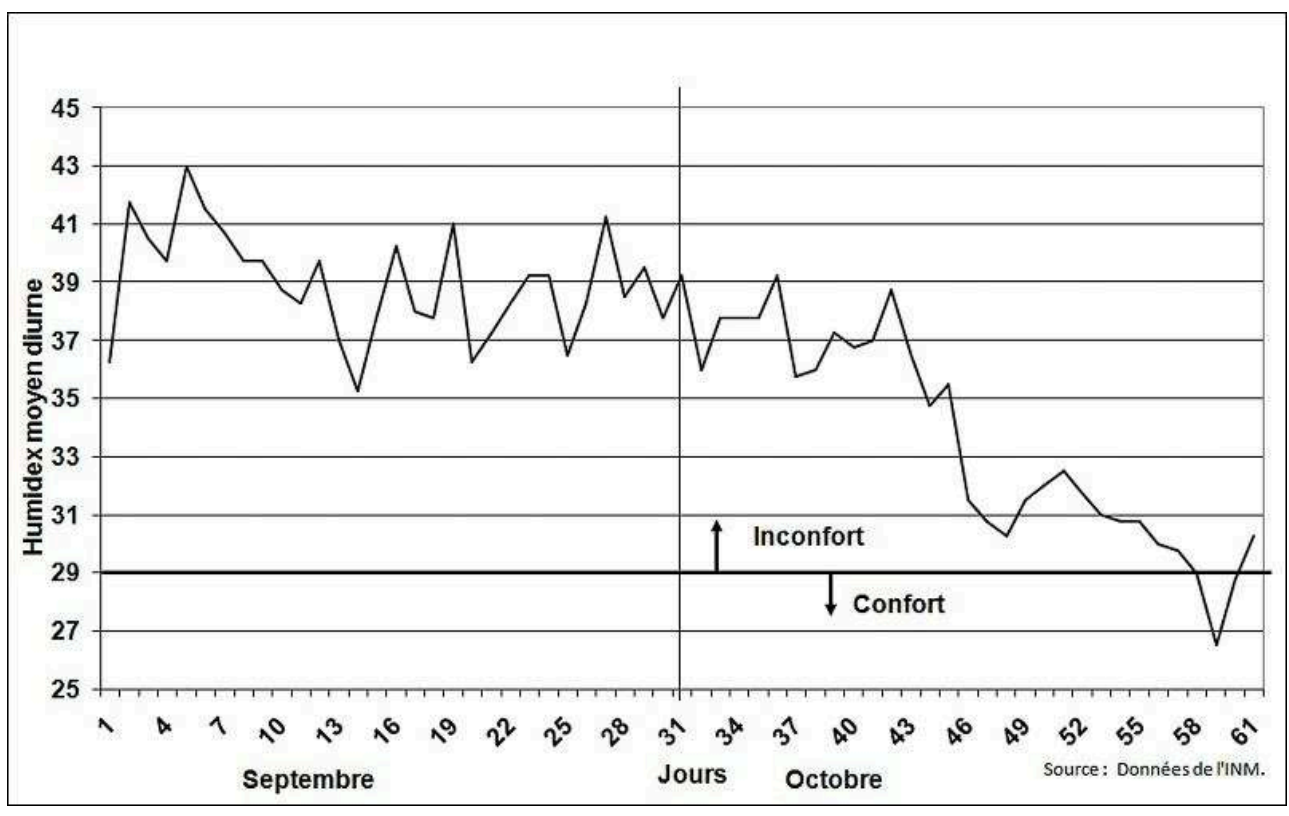

Source : Données de l'INM.

Une thermo-hygrométrie élevée ( $\mathrm{HI} \geq 30$ ) que traduit la prédominance de l'inconfort due à la persistance de la chaleur élevée en automne, s'impose et fragilise d'une part, la santé des gens et renforce d'autre part l'activité des champignons responsables de l'infection et de sa transmission au niveau d'une population vulnérable. Celle-ci regroupe des individus de différents âges, qui peuvent être en bonne santé ou souffrir déjà d'autres maladies (diabète sucré par exemple), ce qui accentue leur fragilité face aux mycoses.

Par ailleurs, la définition des jours à risque et l'étude des aspects météopathologiques qui lui sont associés, durant cette phase à Sfax, permettront d'analyser l'impact de la thermo-hygrométrie automnale dans l'accroissement de la morbidité mycosique.

\section{Définition des jours à risque}

L'analyse de la moyenne diurne de l'indice Humidex (HI) révèle une baisse en septembre, mais surtout en octobre. Si en septembre les HI diurnes sont supérieurs à 37 , en octobre et surtout durant la première moitié du mois ils diminuent à 35 . Cette baisse s'accentue durant la deuxième moitié de ce mois, dans la mesure où les moyennes diurnes de l'indice Humidex (HI) sont inférieures à 31 (illustration 10). En dépit de cette diminution de l'indice Humidex (HI) diurne, l'inconfort thermohygrométrique demeure important, et la situation bioclimatique s'avère favorable pour le développement des champignons responsables des mycoses, qui est expliqué par la transmission inter-humaine accentuée depuis fin d'août. Le pic observé en septembre et les niveaux diurnes élevés de l'indice Humidex (HI) qui dépassent généralement 35 concordent donc. Cette transmission inter-humaine explique la persistance d'une morbidité mycosique élevée en octobre, malgré la diminution des mesures de la thermo-hygrométrie. 
Par ailleurs, la définition des jours à risque est primordiale, afin de déceler l'effet éventuel de l'inconfort sur la fréquence des infections mycosiques durant cette phase (septembre-octobre). Nous suggérons qu'un niveau plus élevé de morbidité mycosique correspond à une augmentation de l'inconfort thermo-hygrométrique. En effet, l'étude des indices Humidex (HI) moyens montre que $93,5 \%$ du nombre total des jours en septembre et octobre sont inconfortables sur la période 2009-2013. Cette part est répartie inégalement entre les quatre modes inconfortables indiqués dans le tableau de l'illustration 3. À l'échelle de la période 2009-2013, les modes exprimant un malaise assez grand $(35<\mathrm{HI}<39)$ et généralisé $(40<\mathrm{HI}<45)$ prédominent, dans la mesure où ces derniers représentent $91,8 \%$ des jours en septembre (illustration 9). Cette prédominance de l'inconfort est attribuée à la fréquence d'un flux maritime tropical de direction Sud-Est (appelé Chlouk) (Ben Boubaker, 1998). Ce flux chaud et humide est à l'origine d'une élévation notable de la thermo-hygrométrie de l'ambiance biométéorologique. Cependant, cette part des jours inconfortables régresse en octobre $(47 \%)$ et le mode exprimant le bien-être $(20<\mathrm{HI}<29)$ représente alors $25 \%$ des jours (illustration 11). En retenant seulement ces deux modes inconfortables cités cidessus, nous remarquons que leur répartition varie d'une année à l'autre. De plus, cette variation inter-annuelle est plus nette en octobre qu'en septembre. Au niveau du mode exprimant un malaise assez grand, la part des jours inconfortables varie de $24,5 \%$ en 2011 à $45,2 \%$ en 2012, alors qu'au niveau du mode exprimant un malaise généralisé, cette part varie de $0 \%$ en 2009 à $25,8 \%$ en 2012 (illustration 11).

Illustration 11 - Variation inter-annuelle de la part des jours exprimant des modes inconfortables en septembre et octobre

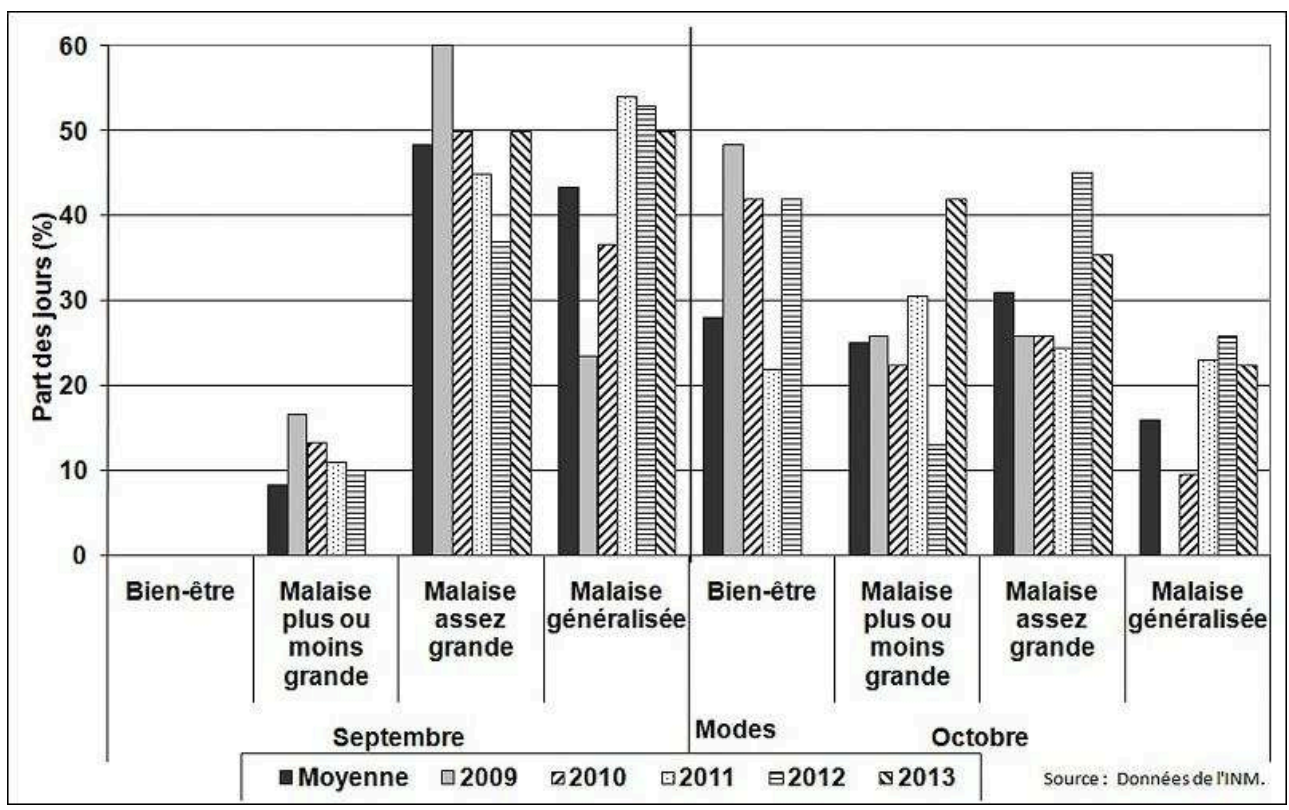

Source : Données de l'INM.

\section{Aspects météopathologiques associés}

Malgré son allègement, perceptible à travers la fréquence du mode exprimant le bienêtre en octobre ( $48,4 \%$ des jours en 2009), l'inconfort reste prédominant puisque les moyennes diurnes de l'indice Humidex (HI) sont supérieures à $29^{\circ} \mathrm{C}$. Cet inconfort 
persistant explique d'une part, l'augmentation notable des consultations mycosiques mensuelles (52\% entre août-septembre) et d'autre part, la variation de celles correspondante aux moyennes de l'indice Humidex (HI) de jours exprimant les deux modes extrêmes retenus: malaise plus ou moins grand et de malaise généralisé. Plusieurs particularités peuvent être identifiées suite à l'analyse de l'évolution de l'état de l'ambiance bioclimatique entre septembre et octobre.

En septembre, la moyenne diurne de l'indice Humidex HI $(33,5)$ des jours durant lesquels s'exprime un malaise plus ou moins grand est inférieure à celle de ceux durant lesquels s'exprime un malaise généralisé $(42,6)$. La relation entre cet inconfort qui augmente et le nombre de consultations pour mycose est nette, puisque celles-ci augmentent de 7 à 18 cas, soit un taux d'accroissement de $157 \%$ (illustration 12). En octobre, les moyennes de l'indice Humidex (HI) diurnes correspondant aux deux modes inconfortables diminuent (de 39,3 pour les jours durant lesquels s'exprime un malaise généralisé à 31 pour les jours durant lesquels s'exprime un malaise plus ou moins grand), en raison de la régression de l'indice Humidex (HI) moyen durant la deuxième moitié de ce mois. Comme la morbidité mycosique diminue en octobre, cela influence celle correspondante aux deux modes inconfortables : l'augmentation est plus ou moins importante en comparaison avec septembre, dans la mesure où les consultations pour mycose augmentent de 8 à 13 cas, soit un accroissement de 62,5\% (illustration 12).

Illustration 12 - Consultations mycosiques en fonction des moyennes de l'indice Humidex HI des jours durant lesquels s'exprime un malaise plus ou moins grand ou généralisé en septembre et octobre

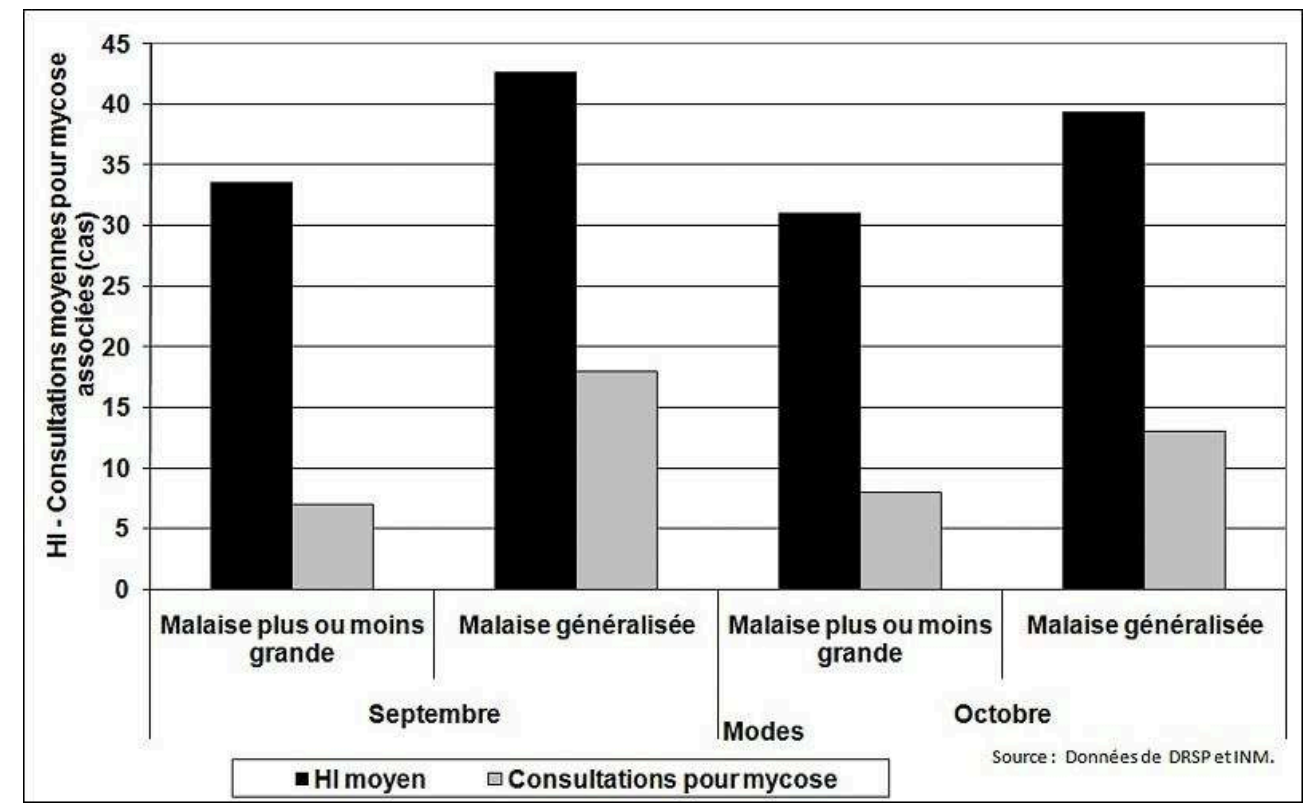

Source: Données de la DRSP et de l'INM

L'ambiance chaude, humide, et donc inconfortable, altère le bien-être et bouleverse l'équilibre thermique entre l'organisme humain et son environnement atmosphérique (Jarraya, 2009). Face à cette situation stressante, l'organisme déclenche la thermolyse pour rétablir son homéothermie. Ce qui provoque une sudation importante. Cependant, les échanges par évaporation de la sueur sont considérablement réduits lorsque le degré hygrométrique de l'air est supérieur à $60 \%$ (Boumaouch, 2008). Une telle situation thermo-hygrométrique renforce l'activité des champignons de dermatophytes 
ou des levures qui affectent la population. L'apport interne en sueur, qui s'intensifie sous une chaleur et humidité élevées, contribue à la fragilisation de l'épiderme par irritation et donc à la multiplication des portes d'entrée pour ces champignons, dont les pathologies se diversifient selon la partie affectée du corps: mycose des pieds, candidoses touchant les fesses et les seins et Herpès Cerciné affectant le visage et les bras... Bien qu'elle régresse, la morbidité mycosique demeure élevée en octobre. En effet, bien que la température de l'air diminue, l'humidité de l'air, élément fondamental de l'ambiance biométéorologique reste importante. Outre la localisation littorale de Sfax, cette humidité automnale élevée s'explique par l'abondance des précipitations en automne, qui représente $70 \%$ des quantités annuelles (Bousnina, 1997). Une ambiance, encore chaude mais surtout plus humide en septembre, favorise l'infection par mycose. Celle-ci accroît au fil des jours et l'aire de la diffusion des champignons s'élargit davantage avec la transmission entre individus (contact direct, mains souillées et utilisation des vêtements et chaussures contaminés).

La diminution de la morbidité mycosique, qui est liée à la régression de l'activité des champignons ralentit le rythme d'accroissement entre les deux modes en octobre. Les facteurs de transmission entre individus indiqués ci-dessus agissent beaucoup plus que l'ambiance inconfortable sur les personnes vulnérables. Ce chiffre en baisse $(62,5 \%)$ indique que la forte activité des champignons - maximale en septembre d'où le pic automnal - atteint sa limite maximale, et explique la diminution de la morbidité mycosique qui a commencé dès octobre (illustration 5). Cette chute des consultations pour mycose signifie que la saison épidémique arrive à terme.

\section{Discussion}

La collecte des informations s'est faite auprès des centres de Santé de Base, ce qui permet de disposer d'une représentativité spatiale puisque ces centres de soins sont répartis dans l'espace étudié (illustration 13). Mais ces structures de soins ambulatoires publiques ne drainent que $60 \%$ de la population de l'agglomération, selon le service des Maladies Communautaires et d'Epidémiologie du Centre Hospitalo-Universitaire Hédi Chaker de Sfax. Les résultats sont donc à généraliser avec prudence, car une partie de la population consulte chez les médecins libéraux. En outre l'automédication réduit aussi l'effectif des personnes consultant les CSB, car les dangers de la mycose, considérée comme une affection banale, sont le plus souvent sous-estimés par les individus affectés. Les données épidémiologiques obtenues n'offrent aucune information sur l'âge des patients et cela représente l'une des limites de notre recherche, qui n'a pas permis de croiser sensibilité aux mycoses et âge des patients. 
Illustration 13 - Répartition des Centres de Santé de Base et localisation géographique du CHU Hédi Chaker dans l'espace étudié

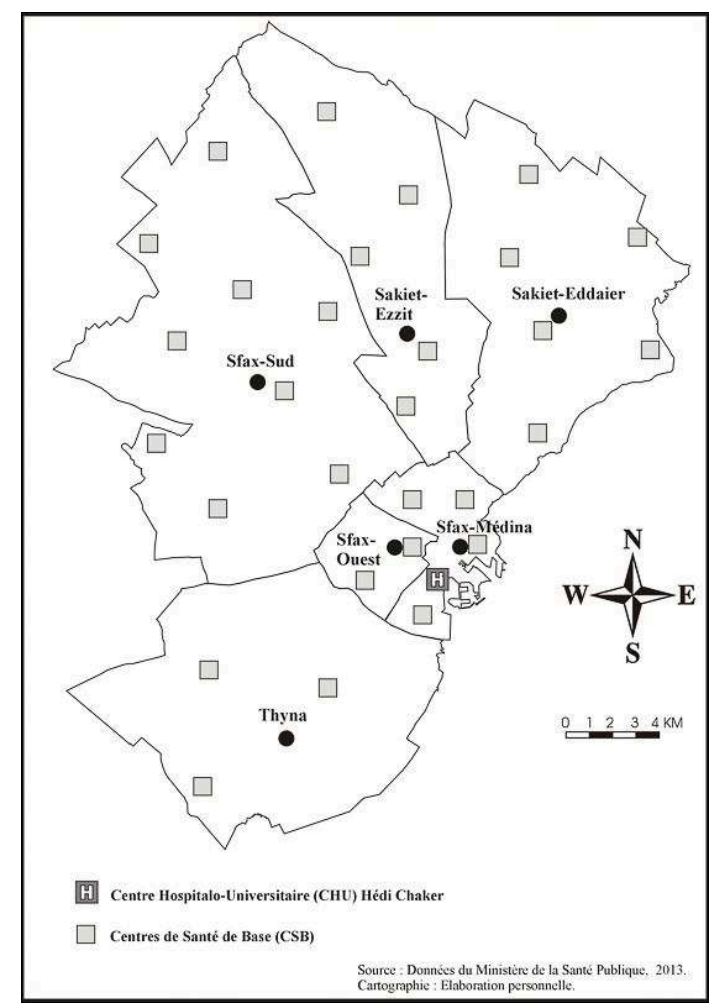

Source : Données du Ministère de la Santé Publique, 2013 ; cartographie : M. Jarraya.

Comme la pyodermite et d'autres infections cutanées non identifiées, la mycose est enregistrée dans la catégorie des maladies infectieuses dans la base de données. Ces données ne fournissent aucune information sur le type de la mycose. C'est pourquoi des données hospitalières identifiant l'infection mycosique ont été intégrées. Cependant, même si elles ont permis de connaître certaines composantes de l'ensemble pathologique de la mycose et de dresser leur régime saisonnier, le pas de temps mensuel de ces informations hospitalières a limité son exploitation dans l'analyse de leur météosensibilité. En l'absence des données journalières par maladie, l'analyse n'a pas pu être conduite plus finement.

Expliquer les deux pics observés à l'échelle mensuelle au printemps et en automne, en mettant en cause le niveau thermo-hygrométrique présent dans l'atmosphère tout en mettant en évidence la proportionnalité des consultations avec l'intensification de la chaleur et de l'humidité était l'objectif de l'étude. Dans ce contexte, la différence est nette entre les deux pics, un pic automnal plus important qu'un pic printanier. Si l'adoucissement thermique printanier associé parfois à une humidité élevée favorise une affection par mycose, la persistance d'une chaleur estivale et surtout une humidité de l'air importante en automne explique une fréquence plus élevée de la morbidité mycosique. C'est pourquoi le pic automnal a été plus particulièrement étudié et a permis de dégager des résultats pertinents. La prédominance des modes thermohygrométriques inconfortables permet d'identifier leurs impacts sur la morbidité mycosique. La forte relation avec la composante thermo-hygrométrique de l'ambiance biométéorologique se manifeste au niveau du degré de confort offert. L'inconfort ne 
traduit pas seulement le stress auquel l'organisme humain doit faire face, mais favorise aussi une activité intense des champignons responsables des infections mycosiques.

L'utilisation de l'indice Humidex, qui exprime la thermo-hygrométrie de l'ambiance biométéorologique, est plus pertinente dans l'analyse du pic automnal que dans celle du pic printanier. En effet, il est plus adapté à l'analyse des ambiances chaudes (Masterson et Richardson, 1979; Steadman, 1984). En outre, la rectification de la fourchette des valeurs exprimant le mode bien-être $(20<\mathrm{HI}<29)$ est nécessaire pour mieux l'adapter au contexte tunisien, car la morbidité mycosique peut varier pendant des jours confortables. Cela confirme le fait que d'autres facteurs étiologiques liés à la maladie elle-même déterminent sa fréquence.

Effectivement, d'autres facteurs interviennent pour expliquer le paysage épidémiologique (Besancenot, 2001 ; 2002). Liés à la présence d'autres maladies comme le diabète ou la défaillance du système immunitaire, ils fragilisent la santé de l'affecté. La vulnérabilité aux mycoses augmente avec la présence de ces maladies et ce sont les personnes âgées, en particulier, qui sont les plus exposées. Par exemple, une glycémie importante chez le diabétique peut favoriser l'infection par les mycoses, car les champignons sont friands de sucre. Un régime alimentaire riche en sucre chez des personnes même non diabétiques, les expose au risque d'infections mycosiques. Les pratiques sociales peuvent aussi favoriser l'exposition des individus à l'infection mycosique. Tout d'abord, les lésions de la peau survenues suite à une action mécanique accidentelle ou à une action chimique, due aux détergents, aux savons trop acides ou au chlore dans les piscines par exemple, constituent une porte d'entrée pour les champignons. Les lésions non ou mal soignées peuvent aboutir à l'apparition des mycoses. Ensuite, l'hygiène hydrique et corporelle ainsi que l'environnement quotidien (l'humidité et aération du logement...) peuvent augmenter le risque d'infection par mycose. En effet, le non-respect des règles d'hygiène corporelle (qualité de l'eau, douche et séchage rigoureux du corps...), l'utilisation des vêtements qui ne permettent pas la transpiration et des chaussures insuffisamment aérées, favorisent le développement des champignons et augmentent le risque d'infections mycosiques. Enfin, la sous-estimation, voire la négligence du risque de contamination entraîne la transmission des mycoses entre individus. L'utilisation d'une serviette commune, des vêtements ou des chaussures d'une personne infectée intensifie le risque d'infection par des mycoses, puisque les champignons se trouvent alors en contact direct avec la peau de la personne saine.

Plusieurs facteurs influencent donc l'infection par les mycoses et favorisent leur propagation au niveau de la population. L'effet des facteurs sociaux cités ci-dessus est permanent - et cela explique la morbidité mycosique durant toute l'année - tandis que la température et l'humidité de l'air jouent le rôle de locomotive dans la hausse saisonnière de la morbidité mycosique au printemps et surtout en automne à Sfax.

\section{Conclusion}

Entre deux pics survenus au printemps et en automne, la morbidité mycosique montre une forte relation avec la thermo-hygrométrie de l'atmosphère. Si la prédominance du confort explique une fréquence plus modérée des consultations pour mycose au printemps, la situation est totalement différente en automne. La thermo-hygrométrie automnale intense, exprimée par des niveaux de l'indice Humidex élevés, explique la 
forte fréquence de la morbidité mycosique. Une relation importante entre l'inconfort thermo-hygrométrique et la morbidité élevée a été identifiée. Compte tenu de l'accroissement notable des consultations pour mycose en relation avec une ambiance biométéorologique qui commence à franchir le seuil de l'inconfort (un malaise plus ou moins grand), il semble que la valeur de l'indice Humidex (HI) moyen de 33,5 puisse être retenue comme seuil au-dessus duquel la morbidité mycosique commence à croître à Sfax, en automne. Les données épidémiologiques recueillies à Sfax indiquent un démarrage de l'épidémie de mycose à partir d'août. Mais une ambiance bioclimatique avec une humidité intense et une chaleur élevée et persistante, conduit à une forte activité des champignons affectant les individus, ce qui explique donc le pic observé au niveau de la morbidité mycosique. En dépit de la diminution de la température de l'air en octobre, la forte humidité continue à régir une thermo-hygrométrie active de l'ambiance et explique donc les niveaux élevés de la morbidité mycosique enregistrés à cette période. Cependant, la transmission active entre individus favorise la contamination maximale de la population vulnérable en septembre et explique aussi l'effectif encore élevé de consultations médicales en octobre. La comparaison de la morbidité associée aux deux modes inconfortables extrêmes montre un relâchement du rythme d'accroissement en octobre. Cela signifie une régression de l'activité des champignons en fin d'épidémie et cela se répercute sur le nombre de consultations, qui baisse en conséquence.

La prise en compte de ces différents aspects de climato pathologie printanière et automnale de la morbidité mycosique à Sfax est primordiale dans la prise en charge de la population vulnérable. Elle se décompose en deux catégories : des individus sains et atteints accidentellement par l'une des infections mycosiques banales ou ceux qui souffrent déjà d'une ou plusieurs maladies sérieuses comme le Syndrome d'Immunodéficience Acquise (SIDA), le diabète sucré ou qui suivent un traitement anticancéreux ou antibiotique... Ces pathologies qui peuvent affecter toutes les parties du corps humain contribuent à altérer fortement la santé d'individus déjà fragilisés par les gênes qu'elles occasionnent comme la démangeaison et l'irritation. Associées à une ambiance chaude et humide, elles gâchent le bien-être des patients infectés et affectent leurs humeurs.

Par ailleurs, il est important de communiquer sur ces aspects climato-pathologiques des mycoses en direction des acteurs de la santé publique à Sfax, afin qu'ils les prennent en compte pendant les deux phases printanière et automnale de la hausse des consultations (Mengmeng et al., 2015). De plus, la prise de conscience des acteurs sanitaires, quels qu'ils soient, de l'éventuelle augmentation de la fréquence des infections mycosiques aussi bien que de plusieurs autres maladies infectieuses sous l'effet du changement climatique (Besancenot, 2007), dont l'extension de la saison chaude à Sfax constitue l'un de ses aspects, les responsabilisent et les impliquent dans la lutte contre les risques sanitaires liés à ce phénomène. Un autre volet autour duquel doit s'articuler la communication est celui de la sensibilisation sanitaire. La diffusion des mesures préventives, basées sur la définition des mycoses, leurs types et les parties $\mathrm{du}$ corps, ciblées au niveau du public et l'urgence de la consultation médicale dès l'apparition des symptômes aident à sensibiliser la population (Jarraya, 2015) et à lutter contre la contamination par les champignons par voie de transmission entre individus. L'hygiène corporelle est aussi un élément fondamental dans la prévention contre les champignons et leurs potentielles complications. La sous-estimation des dangers de cette infection, qui peut représenter une porte d'entrée à d'autres maladies plus 
sérieuses, est susceptible de conduire à une hospitalisation voire à une intervention chirurgicale dans les cas extrêmes.

\section{BIBLIOGRAPHY}

Aurundel A., Sterling E., Biggin J., Sterling TH., 1986. Indirect health effects of relative humidity in indoor environments. Environmental Health Perspectives, n 65, p. 351-361.

Ben Boubaker H., 1998. Le flux de Sud-Est maritime (Chlouk) et ses incidences topothermiques en Tunisie. Publication de l'Association Internationale de Climatologie, $\mathrm{n}^{\circ}$ 11, p. 401-408.

Ben Jemaâ I., 2004. Indicateurs hospitaliers de l'activité de l'EPS Hédi Chaker en 2002. Thèse de doctorat, Université de Sfax, Faculté de Médecine, 73 p.

Ben Salem K., El-Mhamdi S., Letaief M., Bchir M., Soltani M.S., 2011. Epidemiological profile of health care associated infections in the central-east of Tunisia. Eastern Mediterranean Health Journal, Vol. 17, n 6, p. 485-489.

Besancenot J.-P., 2001. Climat et santé. Paris. PUF, Collection médecine et société, 128 p.

Besancenot J.-P., 2002. Vagues de chaleur et mortalité dans les grandes agglomérations urbaines. Environnement Risques Santé, $\mathrm{n}^{\circ}$ 1, p. 229-240.

Besancenot J.-P., 2007. Notre santé à l'épreuve du changement climatique. Paris, Delachaux et Niestlé, $222 \mathrm{p}$.

Besancenot J.-P, Handschumacher P., Ndione J-A., Mbaye I., Laaidi K., 2004. Climat, eau et santé au Sahel ouest-africain. Sécheresse, 15 (3), p. 233-241.

Besancenot J.-P., Thibaudan M., Fonteyne P-A., Nolard N., Berger U., 2011. Changement climatique, moisissures aéroportées et risques sanitaires associés. Convention 2010 DGS / RNSA, $101 \mathrm{p}$.

Bouguerra R., Essaïs O., Sebaï N., Ben Salem L., Amari H., Kammoun M.-R., Chaker E., Zidi B., Ben Slama C., 2004. Prévalence et aspects cliniques des mycoses superficielles chez le diabétique tunisien en milieu hospitalier. Médecine et Maladies Infectieuses, n 34, p. 201-205.

Boumaouch N., 2008. Influence de l'humidité sur le confort thermique intérieur. Actes $d u$ XXIème Colloque de l'Association Internationale de Climatologie, Montpellier 09-13 septembre 2008, p. $145-150$.

Bousnina A., 1997. Le climat de Sfax. Faculté des Sciences Humaines et Sociales Tunis, Ed. Altair, $80 \mathrm{p}$.

Dahech S., 2014. Impact de la brise de mer sur le confort thermique au Maghreb oriental durant la saison chaude. Cybergeo : European Journal of Geography [En ligne], mis en ligne le 02 décembre 2014. URL : http://cybergeo.revues.org/26555; DOI : 10.4000/ cybergeo.26555

Dahech S., Bouaziz R., 2010. Extension de la saison chaude et situation d'inconfort thermique extrême en fin d'été et début d'automne en Tunisie. XXIIIème colloque de l'Association Internationale de Climatologie, Rennes 01-04 septembre, p. 149-154. 
Dowell S., 2001. Seasonal variation in host susceptibility and cycles of certain infectious diseases. Emerging Infectious Diseases, vol. 7, $\mathrm{n}^{\circ}$ 3, p. 369-374.

Dunklin E., Puk TH., 1947. The lethal effect of relative humidity on air-borne bacteria. The department of Medecine and Biochemistry, university of Chicago, p. 87-101.

Edelsberg J., Taneja C., Zervos M., Haque N., Moore C., et al., 2009. Trends in US hospital admissions for skin and soft tissue infections. Emerg Infect Dis., $\mathrm{n}^{\circ}$ 15, p. 1516-1518.

Ehrlich R., Miller S., Walker R., 1970. Relationship between atmospheric temperature and survival of the airborne bacteria. Applied Microbiology, vol. 19, $n^{\circ}$ 2, p. 245-249.

El-Herte R., Baban T., Kanj S., 2012. Mucormycosis : a review on environmental fungal spores and seasonal variation of human disease. Advances in Infectious Diseases, vol. 2, p. 76-81.

Faruqi A.-H., Khan F., Khan K., Haroon T., 1987. Incidence of dermatomycoses in Karatchi in relation to variation in climatic conditions. Journal of the Pakistan Medical Association, vol. 37, $\mathrm{n}^{\circ} 11$, p. 293-295.

Gardner-Medwin J.-M., MacDonald I.-A., Taylor J.-Y., et al., 2001. Seasonal differences in finger skin temperature and microvascular blood flow in healthy men and women are exaggerated in women with primary Raynaud's phenomenon. Br J Clin Pharmacol, n5 52, p. 17-23.

Gentilini M., 1999. Médecine tropicale. Paris, Flammarion, 928 p.

Geyfman M, Andersen B., 2009. How the skin can tell time. J Invest Dermatol, n 129, p. 1063-1066.

Guo-Zheng L., Feng-Feng S., Hao Z., Chun-Pu Z., Hui-Hui L., Jue J., 2015. High mean water vapour pressure promotes the transmission of bacillary dysentery. PLOS ONE, DOI:10.1371/journal.pone. 0124478

Jarraya M., Hammami B., Ghorbel H., Ben Arab N., Maaloul I., Kamoun S., Damak J., Ben Jemaâ M., 2005. Erysipèle des membres inférieurs et thermo-hygrométrie à Sfax en 2004. Poster présenté aux VIIIème Journées Médico-Chirurgicales de Sfax, Faculté de Médecine de Sfax, 15-16 avril 2005.

Jarraya M., 2009. Biométéorologie de la morbidité respiratoire dans le secteur public de la santé à Sfax (Tunisie). Thèse de doctorat, Université Paris Diderot (Paris VII), 364 p.

Jarraya M., 2012. Ambiances froides et consultations respiratoires dans le secteur public de la santé à Sfax (Tunisie). Cybergeo : European Journal of Geography [En ligne], mis en ligne le 30 janvier 2012. URL : http://cybergeo.revues.org/25032 ; DOI : 10.4000/cybergeo.25032

Jarraya M., Beltrando G., 2013. Les services de soins hospitaliers publics dans l'agglomération de Sfax : spécificités fonctionnelles et pouvoir spatial. EchoGéo, mis en ligne le 19 décembre 2013. URL : http://echogeo.revues.org/13632 ; DOI : 10.4000/echogeo.13632.

Jarraya M., 2014. Réchauffement estivo-automnal et fréquence de la diarrhée infantile à Sfax (Centre-Est de la Tunisie). Actes du XXVIIème Colloque de l'Association Internationale de Climatologie, Dijon 02-05 juillet 2014, p. 649-654.

Jarraya M., 2015. Stratégie de communication sanitaire dans un territoire : cas de lutte contre la tuberculose dans le gouvernorat de Sfax en Tunisie. In Merah A. et Meyer V. (dir.), Communication publique et développement territorial : Enjeux d'une valorisation et défis pour les acteurs, p. 129-150.

Klode J., Stoffels I., Körber A., Weindorf M., Dissemond J., 2011. Relationship between the seasonal onset of chronic venous leg ulcers and climatic factors. J Eur Acad Dermatol Venereol, $\mathrm{n}^{\circ} 25$, p. 1415-1419. 
Lakshmipathy D., Kannabiran K., 2010. Review on dermatomycosis: pathogenesis and treatment. Naturel Science, vol. 2, $\mathrm{n}^{\circ}$ 7, p. 726-731.

Masterson J.-M., Richardson F.-A., 1979. Humidex, A Method of Quantifying Human Discomfort Due to Excessive Heat and Humidity. Downsview, Ontario, Environnement Canada, 45 p.

Mengmeng L., Shaohua G., Peng B., Yung Y., Qiyong L., 2015. Heat waves and morbidity: current Knowledge and further direction-A comprehensive literature review. Int. J. Environ. Res. Public Health, $\mathrm{n}^{\circ}$ 12, p. 5256-5283; DOI : 10.3390/ijerph120505256

Morel V., 2013. Vulnérabilité du territoire littoral guyanais aux maladies infectieuses à transmission vectorielle : esquisses de problématiques et perspectives de recherches pluridisciplinaires. EchoGéo, 22, mis en ligne le 30 janvier 2013, consulté le 05 mai 2013. URL : http://echogeo.revues.org/13275 ; DOI : 10.4000/echogeo.13275

Phoenix G., Das S., Joshi M., 2012. Diagnosis and management of cellulitis. BMJ, 345 : e4955.

Piérard-Franchimont C., Piérard G.-E., 2002. Beyond a glimpse at seasonal dry skin. A review. Exog Dermatol, $\mathrm{n}^{\circ}$ 1, p. 3-6.

Piérard-Franchimont C., Hermanns-Lê T., Lesuisse M., Piérard G.-E., 2012. Impact climatique sur les ulcères veineux de jambe. Rev Med Liège, 11, p. 573-575.

Saint-Germain G., 2012. Identification des champignons d'importance médicale. 58 p.

Saad Hussein A., El-Mofty H.M, Hassanien M.A., 2011. Climate change and predicted trend of fungal keratitis in Egypt. Eastern Mediterranean Health Journal, vol. 17, nº 6, p. 468-473.

Simka M., 2010. Seasonal variations in the onset and healing rates of venous leg ulcers. Phlebology, $\mathrm{n}^{\circ} 25$, p. 29-34.

Steadman R.-G., 1984. A universal scale of apparent temperature. Journal of Climate and Applied Meteorology, vol. 23, n 12, p. 1674-1687.

Tang J., 2009. The effect of environmental parameters on the survival of airborne infectious agents. J R Soc Interface, December 6, 6(Suppl 6), S737-S746. DOI : 10.1098/rsif.2009.0227.foc

Xhauflaire-Uhoda E, Mayeux G, Quatresooz P, et al., 2001. Facing up to the imperceptible perspiration. Modulation influences by diabetic neuropathy, physicla excercise and antiperspirant. Skin Res Technol, $\mathrm{n}^{\circ}$ 17, p. 487-493.

http://www.ohcow.on.ca/menuweb/heat_stress_calculator.htm.

http://www.meteo-mussidan.fr/hum.php.

http://www.meteolafleche.com

\section{ABSTRACTS}

The fungal infections are a major public health problem in Sfax (Central-East of Tunisia) because it affects a vulnerable population. The study of the temporal distribution of consultations for mycosis over the period 2009-2013 allowed detecting both spring and falling peaks. The analyzes show a relationship between on the one hand, climate characteristics (temperature and relative humidity rate (heat-humidity)) which establish some discomfort and on the other hand, the increase in cases of fungal infections in the population studied.

Les infections mycosiques constituent un problème majeur de santé publique à Sfax (Centre-Est de la Tunisie), puisqu'elles affectent une population vulnérable. L'étude de la répartition 
temporelle des consultations pour mycose durant la période 2009-2013 a permis de détecter un pic printanier et un pic automnal. Les analyses montrent une relation entre d'une part, les caractéristiques climatiques (température et taux d'humidité relative (thermo-hygrométrie)), qui instaurent un certain inconfort et d'autre part, l'augmentation des cas d'infections mycosiques dans la population étudiée.

\section{INDEX}

Mots-clés: morbidité mycosique, thermo-hygrométrie, pic de consultation, inconfort, Sfax Keywords: mycotic disease, heat-humidity, peak of consultation, discomfort, Sfax

\section{AUTHOR}

\section{MOUNIR JARRAYA}

Mounir Jarraya, jarrayamounir@gmail.com, est enseignant-chercheur en Climatologie à la Faculté des Lettres et Sciences Humaines de Sfax (Université de Sfax) et membre de Laboratoire Syfacte. 